\title{
Análise das possíveis intoxicações decorrentes do uso indiscriminado da ivermectina e hidroxicloroquina durante a pandemia de COVID-19
}

\author{
Analysis of possible intoxications resulting from the indiscriminate use of ivermectin and \\ hydroxychloroquine during the COVID-19 pandemic \\ Análisis de posibles intoxicaciones derivadas del uso indiscriminado de ivermectina e \\ hidroxicloroquina durante la pandemia de COVID-19
}

Recebido: 01/02/2022 | Revisado: 10/02/2022 | Aceito: 10/02/2022 | Publicado: 16/02/2022

\author{
Bárbara Queiroz de Figueiredo \\ ORCID: https://orcid.org/0000-0003-1630-4597 \\ Centro Universitário de Patos de Minas, Brasil \\ E-mail: barbarafigueiredo@unipam.edu.br \\ Bruno Rodrigues Cancela \\ ORCID: https://orcid.org/0000-0002-1348-1398 \\ Universidade Federal do Rio de Janeiro, Brasil \\ E-mail: brcancela@gmail.com \\ Anna Eduarda Linhares Rodrigues \\ ORCID: https://orcid.org/0000-0001-9573-029X \\ Centro Universitário Uninovafapi, Brasil \\ E-mail: annaeduardalr@gmail.com \\ Anna Lira Soares Falcão \\ ORCID: https://orcid.org/0000-0003-1730-4863 \\ Centro Universitário Uninovafapi, Brasil \\ E-mail: annalirafalcao@hotmail.com \\ Diândria Mendonça Martins do Prado \\ ORCID: https://orcid.org/0000-0002-6149-2454 \\ Instituto Master de Ensino Presidente Antônio Carlos, Brasil \\ E-mail: diandriamed@gmail.com \\ Diego da Rocha \\ ORCID: https://orcid.org/0000-0002-3475-2724 \\ Centro Universitário AGES, Brasil \\ E-mail: diegodarocha89@gmail.com \\ Isabelle Carvalho de Melo Lima \\ ORCID: https://orcid.org/0000-0002-1875-9117 \\ Centro Universitário Uninovafapi, Brasil \\ E-mail: isabellemelo.carvalho@gmail.com \\ Raylton Jansen e Silva Segundo \\ ORCID: https://orcid.org/0000-0003-2838-3608 \\ Centro Universitário Uninovafapi, Brasil \\ E-mail: rayltonjansenesilvasegundo@gmail.com
}

\begin{abstract}
Resumo
Introdução: no final de dezembro de 2019, houve um surto de uma doença emergente (COVID-19) devido a um novo coronavírus, chamado SARS-CoV-2, que começou em Wuhan, na China, disseminando-se globalmente como pandemia. O cenário crítico imposto pela tem impulsionado a busca por estratégias farmacológicas terapêuticas e profiláticas, como a ivermectina e hidroxicloroquina. Objetivo: evidenciar, por meio de análises empíricas e atuais, os riscos associados ao uso indiscriminado de ivermectina e hidroxicloroquina como terapêutica contra a COVID19, ressaltando os efeitos de toxicidade destes medicamentos. Metodologia: trata-se de uma pesquisa descritiva do tipo revisão integrativa da literatura. A pesquisa foi realizada através do acesso online nas bases de dados National Library of Medicine (PubMed MEDLINE), Scientific Electronic Library Online (Scielo), Cochrane Database of Systematic Reviews (CDSR), Google Scholar, Biblioteca Virtual em Saúde (BVS) e EBSCO Information Services, no mês de agosto 2021. Resultados e discussão: de acordo com a ANVISA, ainda não há estudos conclusivos que aceitem a hipótese de que a ivermectina apresente eficácia no tratamento da COVID-19. Já a hidroxicloroquina, em relação ao seu mecanismo de ação, acredita-se que a hidroxicloroquina aja, sob infecção por COVID-19, impedindo a glicosilação terminal da enzima ACE2, que, por sua vez, atua como receptor de membrana para o SARS-CoV-2, podendo, então, impedir a ligação do vírus. No entanto, ainda não há contestação sobre a eficácia desses medicamentos sob a COVID-19, e, inclusive, há relatos de toxicidade orgânica relacionada ao uso indiscriminado
\end{abstract}


destes medicamentos, associados a problemáticas como hemorragias, insuficiência renal, arritmias e até mesmo hepatite medicamentosa. Considerações finais: é de suma importância o papel do médico e, mais ainda, do farmacêutico clínico, que vai além do acompanhamento farmacoterapêutico a pacientes que fazem uso da polifarmácia.

Palavras-chave: Ivermectina; Hidroxicloroquina; COVID-19; Intoxicação.

\begin{abstract}
Introduction: In late December 2019, there was an outbreak of an emerging disease (COVID-19) due to a new coronavirus, called SARS-CoV-2, which started in Wuhan, China, spreading globally as a pandemic. The critical scenario imposed by has driven the search for therapeutic and prophylactic pharmacological strategies, such as ivermectin and hydroxychloroquine. Objective: to highlight, through empirical and current analyses, the risks associated with the indiscriminate use of ivermectin and hydroxychloroquine as therapeutics against COVID-19, highlighting the toxicity effects of these drugs. Methodology: this is a descriptive research of the integrative literature review type. The research was carried out through online access to the National Library of Medicine (PubMed MEDLINE), Scientific Electronic Library Online (Scielo), Cochrane Database of Systematic Reviews (CDSR), Google Scholar, Virtual Health Library (BVS) and EBSCO databases. Information Services, in August 2021. Results and discussion: according to ANVISA, there are still no conclusive studies that accept the hypothesis that ivermectin is effective in the treatment of COVID-19. As for hydroxychloroquine, in relation to its mechanism of action, hydroxychloroquine is believed to act, under COVID-19 infection, by preventing terminal glycosylation of the ACE2 enzyme, which, in turn, acts as a membrane receptor for SARS- CoV-2, which can then prevent the virus from binding. However, there is still no dispute about the effectiveness of these drugs under COVID-19, and there are even reports of organic toxicity related to the indiscriminate use of these drugs, associated with problems such as bleeding, renal failure, arrhythmias and even drug hepatitis. Final considerations: the role of the physician and, even more, the clinical pharmacist, is of paramount importance, which goes beyond the pharmacotherapeutic follow-up to patients who use polypharmacy.
\end{abstract}

Keywords: Ivermectin; Hydroxychloroquine; COVID-19; Intoxication.

\begin{abstract}
Resumen
Introducción: A fines de diciembre de 2019, hubo un brote de una enfermedad emergente (COVID-19) debido a un nuevo coronavirus, llamado SARS-CoV-2, que comenzó en Wuhan, China, propagándose globalmente como una pandemia. El escenario crítico impuesto por ha impulsado la búsqueda de estrategias farmacológicas terapéuticas y profilácticas, como la ivermectina y la hidroxicloroquina. Objetivo: destacar, a través de análisis empíricos y actuales, los riesgos asociados al uso indiscriminado de ivermectina e hidroxicloroquina como terapéutica contra la COVID-19, destacando los efectos de toxicidad de estos fármacos. Metodología: se trata de una investigación descriptiva del tipo revisión integrativa de la literatura. La investigación se llevó a cabo a través del acceso en línea a la Biblioteca Nacional de Medicina (PubMed MEDLINE), Scientific Electronic Library Online (Scielo), Cochrane Database of Systematic Reviews (CDSR), Google Scholar, Virtual Health Library (BVS) y las bases de datos EBSCO. Servicios, en agosto de 2021. Resultados y discusión: según la ANVISA, aún no existen estudios concluyentes que acepten la hipótesis de que la ivermectina es efectiva en el tratamiento de la COVID-19. En cuanto a la hidroxicloroquina, en relación a su mecanismo de acción, se cree que la hidroxicloroquina actúa, bajo infección por COVID-19, impidiendo la glicosilación terminal de la enzima ACE2, que, a su vez, actúa como receptor de membrana para el SARS-CoV-2, que luego puede evitar que el virus se una. Sin embargo, aún no hay controversia sobre la efectividad de estos medicamentos bajo el COVID-19, e incluso hay reportes de toxicidad orgánica relacionada con el uso indiscriminado de estos medicamentos, asociada a problemas como hemorragias, insuficiencia renal, arritmias e incluso hepatitis medicamentosas. Consideraciones finales: el papel del médico y, más aún, del farmacéutico clínico, es de suma importancia, que va más allá del seguimiento farmacoterapéutico a los pacientes que utilizan polifarmacia.
\end{abstract}

Palabras clave: Ivermectina; Hidroxicloroquina; COVID-19; Intoxicación.

\title{
1. Introdução
}

No final de dezembro de 2019, houve um surto de uma doença emergente (COVID-19) devido a um novo coronavírus, chamado SARS-CoV-2, que começou em Wuhan, na China, disseminando-se globalmente como pandemia. O cenário crítico imposto pela tem impulsionado a busca por estratégias farmacológicas terapêuticas e profiláticas. Entre os potenciais medicamentos ao tratamento da COVID-19, está o reposicionamento de fármacos antigos para uso como tratamento antiviral, por ter um melhor conhecimento sobre perfil de segurança, efeitos colaterais, posologia e interações medicamentosa, dentre elas, a ivermectina e a hidroxicloroquina, medicações preconizadas pelo chamado "Kit Covid” (Pacheco et al. 2020). 
A ivermectina é um agente antiparasitário pertencente ao grupo das avermectinas, originalmente um metabólito de fermentação produzido pela bactéria Streptomyces avermitilis. Foi introduzida para uso médico em 1982 e é eficaz contra vários tipos de nematelmintos e helmintos e ectoparasitas, como ácaros e piolhos. O modo de ação é baseado na ligação a canais específicos da membrana celular que ocorrem apenas em invertebrados. A ativação do canal leva ao bloqueio da transmissão do sinal celular por meio da hiperpolarização induzida por cloreto. Consequentemente, os parasitas são paralisados e morrem, interrompendo seu ciclo de reprodução (Melo et al. 2020).

Em animais e humanos, a ivermectina é facilmente reabsorvida pela mucosa se tomada por via oral ou pela pele se tomada por via tópica. Como um composto lipofílico, ele se acumula na gordura e no tecido do fígado, de onde se espalha e tem efeito. A eliminação é processada por meio da bile e das fezes. A ivermectina é amplamente utilizada na medicina veterinária, mas também é aprovada para doenças parasitárias humanas, como oncocercose, filariose linfática, estrongiloidíase e sarna em vários países. Atualmente, o grande interesse global em relação à ivermectina recai sobre o seu potencial no tratamento da COVID-19 (Pedroso et al. 2020).

Já a hidroxicloroquina, um análogo da cloroquina, usado como antimalárico e com propriedades anti-inflamatórias, apresenta ação esquizonticida e gametocida, mas como imunossupressora ainda não tem seu mecanismo totalmente elucidado, acredita-se que ela suprima o processamento de antígeno intracelular e o carregamento de peptídios nas moléculas MHC de classe ao aumentar o pH dos compartimentos lisossomal e endossomal, diminuindo, assim, a ativação da célula $\mathrm{T}$ e também há diminuição da quimiotaxia dos leucócitos, estabilização das enzimas lisossomais, inibição da síntese de DNA e RNA e captação de radicais livres (Lacava, 2010). Em relação ao seu mecanismo de ação, acredita-se que a hidroxicloroquina aja, sob infecção por COVID-19, impedindo a glicosilação terminal da enzima ACE2, que, por sua vez, atua como receptor de membrana para o SARS-CoV-2, podendo, então, impedir a ligação do vírus (Fantini, 2020). Desse modo, o presente estudo tem, como objetivo, evidenciar, por meio de análises empíricas e atuais, os riscos associados ao uso indiscriminado de ivermectina e hidroxicloroquina como terapêutica contra a COVID-19, ressaltando os efeitos de toxicidade destes medicamentos.

\section{Metodologia}

Trata-se de uma pesquisa descritiva do tipo revisão integrativa da literatura, que buscou evidenciar, por meio de análises empíricas e atuais, os riscos associados ao uso indiscriminado de ivermectina e hidroxicloroquina como terapêutica contra a COVID-19, ressaltando os efeitos de toxicidade destes medicamentos. A pesquisa foi realizada através do acesso online nas bases de dados National Library of Medicine (PubMed MEDLINE), Scientific Electronic Library Online (Scielo), Cochrane Database of Systematic Reviews (CDSR), Google Scholar, Biblioteca Virtual em Saúde (BVS) e EBSCO Information Services, no mês de agosto 2021. Para a busca das obras foram utilizadas as palavras-chaves presentes nos descritores em Ciências da Saúde (DeCS): em inglês: "ivermectin", "hydroxychloroquine", "COVID-19", "intoxication", "adverse effects" e em português: "ivermectina", "hidroxicloroquina", "COVID-19", "intoxicação", "efeitos adversos".

Como critérios de inclusão, foram considerados artigos originais, que abordassem o tema pesquisado e permitissem acesso integral ao conteúdo do estudo, publicados no período de 2015 a 2021, em inglês e português. O critério de exclusão foi imposto naqueles trabalhos que não estavam em inglês ou português, que não tinham passado por processo de Peer-View e que não abordassem o tema da pesquisa. A estratégia de seleção dos artigos seguiu as seguintes etapas: busca nas bases de dados selecionadas; leitura dos títulos de todos os artigos encontrados e exclusão daqueles que não abordavam o assunto; leitura crítica dos resumos dos artigos e leitura na íntegra dos artigos selecionados nas etapas anteriores. Assim, totalizaram-se 28 artigos científicos para a revisão integrativa da literatura, com os descritores apresentados acima. 


\section{Resultados e Discussão}

\subsection{Ivermectina}

A ivermectina, uma droga usada para tratar parasitas como sarna em humanos e helmintos intestinais em bovinos, foi testada em 2020 como terapêutica contra COVID-19. No entanto, os testes de laboratório sugeriram um efeito fraco sobre o vírus SARS-CoV-2 em um tubo de ensaio, mas não parecia viável em humanos, pois as doses necessárias seriam grandes. Entretanto, mesmo com as frágeis evidências de eficácia da ivermectina clinicamente contra a COVID-19, pequenos testes iniciais sugeriram grandes efeitos sobre a mortalidade, e isso levou alguns grupos de defesa a fazerem "lobby" para sua introdução generalizada em todo o mundo (Popp et al. 2021).

Sob essa perspectiva, no Brasil, as buscas por ivermectina cresceram aproximadamente 5300\% apenas em 2020, segundo dados da plataforma brasileira "Consulta Remédios". Esse aumento alarmante na venda da medicação está principalmente associado à não necessidade de receita médica, o que fez com que esse vermífugo se tornasse o novo alvo da automedicação na tentativa de prevenção contra a COVID-19. O aumento da procura pela ivermectina foi tão grande que ela passou a ser considerada a "nova cloroquina", o que levou a Agência Nacional de Vigilância Sanitária (ANVISA) a incluí-la na categoria de medicamentos submetidos a controle especial no dia 23 de Julho de 2020 (Lima et al. 2020).

Apesar do agente antiparasitário de amplo espectro ter demonstrado atividade in vitro, impedindo a replicação viral do SARS-CoV-2, Caly et al. (2020) demonstraram que a dose necessária para se alcançar a concentração plasmática à atividade antiviral em humanos é mais de cem vezes a dose usual. Desse modo, o efeito terapêutico é condicionado à utilização de grandes quantidades desse fármaco, o que certamente corrobora problemáticas no que se refere à segurança dessa terapia.

Segundo González et al. (2021), seguindo as doses recomendadas, a ivermectina é geralmente bem tolerada. Os efeitos adversos, que parecem surgir parcialmente da morte rápida dos parasitas, levando à hiperinflamação e reações anafiláticas, incluem fraqueza, sonolência, diarreia, náuseas e vômitos. Além disso, a ivermectina pode causar febre e erupção cutânea. Todavia, podem ocorrer efeitos adversos graves raros, como problemas de visão, neurotoxicidade e danos ao fígado. De acordo com a ANVISA, ainda não há estudos conclusivos que aceitem a hipótese de que a ivermectina apresente eficácia no tratamento da COVID-19. Eleger a ivermectina para este tratamento é de responsabilidade do médico prescritor (ANVISA, 2020).

Apesar de parecer segura e bem tolerada (Patrì et al. 2020). deve-se considerar a relação risco versus benefício no uso da droga, uma vez que os efeitos colaterais não podem ser negligenciados, que incluem: reações dermatológicas, gastrointestinais, neuromusculares e cardiovasculares. Portanto, o seu uso em pacientes com patologias que acometem o trato gastrointestinal, sistema nervoso, sistema muscular e sistema cardiovascular devem ser cuidadosamente analisado (Chaves, 2020). Recentemente, o médico pneumologista e presidente da Sociedade Paulista de Pneumologia e Tisiologia (SPPT), Frederico Fernandes, revelou em seu perfil no Twitter o caso de uma paciente que contraiu hepatite medicamentosa devido ao uso de ivermectina no tratamento contra a COVID-19, com dosagem de 18mg por dia durante uma semana, e devido às lesões graves, foi realizado o transplante de fígado.

\subsection{Hidroxicloroquina}

A hidroxicloroquina (HCQ) é um fármaco antimalárico com propriedades anti-inflamatórias, utilizado, também, como imunomodulador em casos de doenças reumatológicas, como lúpus eritematoso sistêmico, estando facilmente acessível à população, favorecendo o abuso de seu uso, mesmo sob obrigatoriedade de receita médica (Batista et al. 2021). No início da pandemia, face à indefinição da história natural da doença e da grande quantidade de pacientes graves em insuficiência respiratória aguda, o desespero em proporcionar um tratamento que modificasse o curso da doença levou a uma série de medidas compassivas (Saag 2020). 
Em março de 2020, um estudo com qualidade metodológica extremamente limitada sugeria benefício clínico da hidroxicloroquina (Gautret et al. 2020), sendo, posteriormente, retratado pelo próprio autor. O entusiasmo inicial foi empregado politicamente, porém já em junho de 2020, a agência Food and Drug Administration (FDA) revogou seu uso após a publicação de dados preliminares de um grande estudo multicêntrico conduzido no Reino Unido em pacientes hospitalizados (Gautret et al. 2021). Alguns meses depois, a Organização Mundial da Saúde (OMS) também publicou os dados de seu grande estudo adaptativo multicêntrico, corroborando a conclusão de completa ausência de eficácia da hidroxicloroquina na mesma população, tanto com relação à mortalidade como a necessidade de instalação de ventilação mecânica ou a tempo de hospitalização (WHO, 2021).

Pouquíssimos fármacos foram tão escrutinados e explorados quanto a potencial terapêutico como cloroquina ou hidroxicloroquina na COVID-19 (Singh et al. 2021). Nenhum estudo de intervenção, randomizado, demonstrou benefício clínico da cloroquina ou hidroxicloroquina na abordagem da COVID-19 em pacientes ambulatoriais ou hospitalizados, seja na prevenção ou no tratamento, mesmo quando incluídos trabalhos não publicados (Axfors et al. 2021). A alegação de que o benefício só seria demonstrado em pacientes ainda em fase leve da doença não foi confirmada (Cavalcanti et al. 2020), tampouco em momento definitivamente precoce, antes do aparecimento de sintomas (Mitjà et al. 2021). Adicionalmente, o compilado de evidências sugere que o uso de cloroquina e/ou hidroxicloroquina está associado a incremento de mortalidade devido à incidência de eventos adversos graves (Boulware et al. 2020).

Aliado a isso, Ferner et al (2020) sinalizam quanto aos eventos adversos, alegando que o uso amplo da hidroxicloroquina pode expor os pacientes a consequências fatais, incluindo reações cutâneas, falência hepática e arritmia ventricular, principalmente se prescrita associada a azitromicina. Segundo estudo de Duan et al. (2020), as intoxicações por HCQ são episódios pouco frequentes, apesar de sua ampla utilização no tratamento de desordens reumatológicas (Merino et al. 2017). Embora rara, a overdose por HCQ acarreta riscos consideráveis de morbimortalidade (Olano et al. 2019).

O medicamento apresenta rápida absorção e atinge pico de concentração em até duas horas após sua ingestão, podendo, assim, diante de uma sobredosagem, ocasionar a morte em até três horas (Broeke et al. 2016). Nesse contexto, o início dos sintomas de intoxicação podem aparecer durante os primeiros 30 minutos, principalmente pela característica de fácil absorção pelos tecidos com um grande volume de distribuição, justificando o longo período de meia vida que pode chegar até a 40 dias para excreção de grandes dosagens, e afirma que altas doses da droga é capaz de interferir no efluxo de potássio e na liberação de insulina, assemelhando-se com o mecanismo de intoxicação causado pela cloroquina (Mcbeth et al. 2015).

Nesse contexto, Olano (2019) destaca que o pico de concentração plasmática entre 3 a 12 horas e a alta capacidade de adsorção por carvão ativado também são características comuns entre a hidroxicloroquina e cloroquina, além de enfatizar que a metabolização da hidroxicloroquina ocorre principalmente no fígado, justificando a hepatoxicidade apresentada em alguns casos de abuso da droga. Ademais, a toxicidade da hidroxicloroquina está primariamente relacionada a complicações cardiovasculares. Por meio da sua propriedade de ligação aos canais de sódio, ocasiona um bloqueio semelhante ao observado por drogas antiarrítmicas de classe I, promovendo retardo na condução ventricular e possibilitando a ocorrência de arritmias (Broeke et al. 2016).

A hidroxicloroquina pode causar toxicidade retiniana, de modo assintomático. Os sintomas são indicativos de severidade. Um dos fatores mais importantes aparenta ser a dosagem e o efeito cumulativo da hidroxicloroquina. E o risco de toxicidade é maior em pacientes obesos devido à clearance do medicamento. Algumas contraindicações são: a dosagem diária recomendada não exceder 400mg, fármaco não deve ser usado por mais de 5 anos, doença renal ou hepática concomitante, doença retiniana ou maculopatia, idade maior que 60 anos (Zhou et al. 2020).

Doses de hidroxicloroquina maiores que 300mg atingem pico de níveis plasmáticos no intervalo de 1-6 horas. A eliminação de hidroxicloroquina é lenta e pode ser detectada na urina durante várias semanas. Cerca de $30 \%$ do fármaco foram 
eliminados pelo fígado, e o restante é excretado na urina. Possui meia-vida longa: cerca de 1 mês e leva aproximadamente seis meses para atingir eliminação completa no corpo, e tal fato deve ser levado em consideração nos casos de toxicidade. A hidroxicloroquina pode desencadear ataque agudo com febre e elevação de enzimas hepáticas, sendo necessário fazer controle dos níveis de bilirrubina e atentar para sinais de toxicidade (Menezes et al. 2020).

Outrossim, estudo de Chen et al. (2020) apresentou como efeitos adversos diarreia transitória e elevação anormal da função hepática com uso de sulfato hidroxicloroquina, e abordou a necessidade de uso de hidroxicloroquina enquanto escassez de fármacos para tratamento específico, da doença COVID-19, porém relatou que o fármaco, por seu intenso efeito causador de retinopatia, distúrbios de condução cardiológicos e sintomas neurológicos, deve ser mais bem explorado por efeitos de overdose antes da distribuição em larga escala (Chen et al. 2020) O estudo de Barbosa et al. (2020) observou como efeito adverso da hidroxicloroquina o desenvolvimento de arritmia do tipo "torsades de pointes" e o estudo de Borba et al. (2020) demonstrou desenvolvimento de miocardite, rabdmiólise e prolongamento do segmento QT (não especificando se houve arritmia) com o uso da cloroquina.

A intoxicação por HCQ é potencialmente letal e ainda rara mundialmente, e a gravidade do quadro de intoxicação deve-se, principalmente, à toxicidade cardiovascular e às suas implicações clínicas, levando o paciente, muitas vezes, a prognósticos reservados (Broeke et al. 2016). A absorção rápida da hidroxicloroquina pelo organismo possibilita picos de concentração sérica elevados logo após sua ingestão exercendo efeitos tóxicos no sistema de condução cardíaco, bem como no próprio miocárdio, além de efeitos inotrópicos negativos (Merino et al. 2017).

\section{Considerações Finais}

Portanto, nota-se que o "Kit Covid", principalmente quando tratando-se da ivermectina e hidroxicloroquina, podem ser potencialmente tóxicos ao organismo humano, haja vista as correlações entre os efeitos adversos e relatos de casos clínicos ressaltando, de maneira empírica, essa problemática. Além disso, a hepatite medicamentosa que foi relatada recentemente, resultante de altas doses de ivermectina, é uma observação bastante preocupante sob o âmbito atual, haja vista, também, a apresentação de problemas como hemorragias, insuficiência renal e arritmias. Desse modo, é de suma importância o papel do médico e, mais ainda, do farmacêutico clínico, que vai além do acompanhamento farmacoterapêutico a pacientes que fazem uso da polifarmácia. Além disso, compete aos profissionais, também, repassar orientações sobre as medidas corretas e necessárias para a prevenção contra a COVID-19, e, dessa maneira, educar os pacientes sobre conhecimentos, até então, ilustrados e comprovados pela ciência.

\section{Referências}

Axfors, C., et al. (2021). Mortality outcomes with hydroxychloroquine and chloroquine in COVID-19 from an international collaborative meta-analysis of randomized trials. Nat Commun, 12 (2349), 1-9.

Barbosa, J., et al. (2020). Outcomes of Hydroxychloroquine in Hospitalized Patients with COVID-19: A Quasi-Randomized Comparative Study. N Engl J Med, 7 (6), 21-25.

Batista T. A. B., et al. (2021). Clinical implications and cardiotoxicity in hydroxychloroquine overdose. Revinter, 14 (2), 29-35.

Borba, M. G. S., et al. (2020). Chloroquine diphosphate in two different dosages as adjunctive therapy of hospitalized patients with severe respiratory syndrome in the context of coronavirus (SARS-CoV-2) infection: Preliminary safety results of a randomized, double-blinded, phase IIb clinical trial (CloroCOVID-19 Study). MedRxiv.

Brasil. Agência Nacional de Vigilância Sanitária (ANVISA). Nota de esclarecimento sobre a ivermectina. 2020.

Broeke, R. T., et al. (2016). Early treatment with intravenous lipid emulsion in a potentially lethal hydroxychloroquine intoxication. Neth J Med, 74 (5), 210214.

Caly, L., et al. (2020). O medicamento aprovado pela FDA ivermectina inibe a replicação do SARS-CoV-2 in vitro. Elsevier, 178 (104), 1-7. 
Cavalcanti, A. B., et al. (2020). Hydroxychloroquine with or without Azithromycin in Mild-to-Moderate COVID-19. N Engl J Med, 383 (11), 2041-2052.

Chaves, E.F. et al. (2020). Considerações sobre os medicamentos com potencial efeito farmacológico para o vírus SARS-HCoV-2. Departamento de Farmácia - AMIB.

Chen, J., et al. (2020). A pilot study of hydroxychloroquine in treatment of patients with common coronavirus disease-19 (COVID-19). J Zhejiang Univ (Med Sci), 49 (2), 24-31.

Chen, Z., et al. (2020). Efficacy of hydroxychloroquine in patients with COVID-19: results of a randomized clinical trial. MedRxiv.

Duan, Y. J., et al. (2020). The Trial of Chloroquine in the Treatment of Corona Virus Disease 2019 (COVID-19) and Its Research Progress in Forensic Toxicology. Fa Yi Xue Za Zhi, 36 (2), 34-38.

Fantini J., et al. (2020). Estudos de modelagem estrutural e molecular revelam um novo mecanismo de ação da cloroquina e hidroxicloroquina contra a infecção por SARS-CoV-2. International Journal of Antimicrobial Agents, 1 (8), 56-63.

Ferner, R. E., et al. (2020). Chloroquine and hydroxychloroquine in COVID-19. BMJ, 369 (1432), 1-7.

Gautret, P., et al. (2021). Effect of hydroxychloroquine and azithromycin as a treatment of COVID-19: results of an open-label non-randomized clinical trial, an update with an intention-to-treat analysis and clinical outcomes. Int J Antimicrob Agents, 57 (1).

Gautret P., et al. (2020). Hydroxychloroquine and azithromycin as a treatment of COVID-19: results of an open-label non-randomized clinical trial. Int J Antimicrob Agents, 56 (1), 724-731.

Gonzalez, B. J., et al. (2021). Eficácia e segurança de ivermectina e hidroxicloroquina em pacientes com COVID-19 grave. Um ensaio clínico randomizado. Medrix.

Lacava, A. C. (2010). Complicações oculares da terapêutica com a cloroquina e derivados. Arquivos Brasileiros de Oftalmologia, 73 (4), $384-389$.

Lima, W. G., et al. (2020). Uso irracional de medicamentos e plantas medicinais contra a COVID-19 (SARS-CoV-2): Um problema emergente. Brazilian Journal of Health and Pharmacy, 2 (3), 37-53.

Mcbeth, P. B., et al. (2015). Novel therapies for myocardial irritability following extreme hydroxychloroquine toxicity. Case Reports in Emergency Medicine, 87 (6), 1-6.

Melo, P. A., et al. (2020). Do ponto de vista farmacológico, o uso da ivermectina poderia ser eficaz frente à infecção por COVID-19? Uma revisão bibliográfica. Almanaque Multidisciplinar de Pesquisa, 7 (2), 98-100.

Menezes C. R., et al. (2020). Effectiveness and toxicity of chloroquine and hydroxychloroquine associated (or not) with azithromycin for the treatment of COVID-19. What do we know so far? J. Health Biol Sci., 8 (1), 1-9.

Merino, A., et al. (2017). Hydroxychloroquine, a potentially lethal drug. Medicina Intensiva, 41 (4).

Mitjà, O., et al. (2021). A Cluster-Randomized Trial of Hydroxychloroquine for Prevention of COVID-19. N Engl J Med, 384 (2), $417-427$.

Olano, J., et al. (2019). Toxicokinetics of hydroxychloroquine following a massive overdose. The American Journal of Emergency Medicine, 37 (12).

Pacheco, T. J. A., et al. (2020). Panorama mundial de estudos com a hidroxicloroquina para o tratamento da COVID-19. Journal of Health \& Biological Sciences, 8 (1), 34-41.

Patrì, A., et al. (2020). Hydroxychloroquine and ivermectin: A synergistic combination for COVID-19 chemoprophylaxis and treatment? Journal of the American Academy of Dermatology, 82 (6).

Pedroso, L. A., et al. (2020). Aspectos farmacológicos da ivermectina e seu potencial uso no tratamento da COVID-19. Brazilian Journal of Health and Pharmacy, 2 (3), 76-81.

Popp, M., et al. (2021). Ivermectina para prevenir e tratar COVID - 19. Cochrane Database of Systematic Reviews, 7 (1), 87-91.

Saag, M. S., et al. (2020). Misguided Use of Hydroxychloroquine for COVID-19: The Infusion of Politics into Science. JAMA, 324 (1), $2161-2162$.

Singh, B., et al. (2021). Chloroquine or hydroxychloroquine for prevention and treatment of COVID-19. Cochrane Database Syst Rev, 2 (13587), 43-47.

WHO Solidarity Trial Consortium. (2021). Repurposed Antiviral Drugs for COVID-19 - Interim WHO Solidarity Trial Results. N Engl J Med, 384 (87), $497-$ 511.

Zhou, D., et al. (2020). COVID-19: a recommendation to examine the effect of hydroxychloroquine in preventing infection and progression, $J$ Antimicr Chemotherapy, 75 (7), 1667-1670. 